\title{
A NEW INJURY OF THE DISTAL RADIO-ULNAR JOINT
}

\author{
H. K. GRAHAM, G. F. McCOY, R. A. B. MOLLAN
}

From the Royal Victoria Hospital, Belfast

\begin{abstract}
A case of locked hypersupination of the distal radio-ulnar joint is described in which the ulnar displacement was purely rotational rather than volar. The mechanism of injury is discussed in the light of cadaveric dissections which reproduced the injury.
\end{abstract}

Injury of the distal radio-ulnar joint is well recognised in association with the eponymous forearm fractures of Colles, Smith, and Galleazzi (Vesely 1967; Dobyns and Linscheid 1975). However, in the absence of a fracture, isolated injury to the distal radio-ulnar joint is often unrecognised, or is misdiagnosed as a "sprained wrist"; the resulting inadequate treatment may lead to prolonged disability (Wright 1980).

Simple volar dislocation of the ulna was first described by Sir Astley Cooper in 1822 (Gibson 1925). Since then, there have been a number of reports of both dorsal and volar dislocation with suggestions as to the mechanisms of injury (Pezeshki and Weiland 1978; Morrissy and Nalebuff 1979). We report a purely rotational injury to the distal radio-ulnar joint, in which there was no displacement of the ulna in a dorsal or volar direction. This injury, as far as we know, has not been described before.

\section{CASE REPORT}

A 52-year-old electrical engineer was carrying a television set when he tripped, causing the set to slip from his grasp. This resulted in forced hypersupination of his outstretched right hand, and he presented complaining of a "locked wrist" associated with severe pain. On examination, the forearm and hand were held in 180 of fixed hypersupination. Mild swelling of the wrist was seen, but the distal ulna was not unduly prominent.

A postero-anterior radiograph, taken in the hypersupinated position of deformity, showed an unusual appearance: the ulnar styloid was lying in the sigmoid notch of the radius; lateral displacement was only a few millimetres more than the normal amount in full supination (Fig. 1), but there was 90 more supination than is normally possible. A true lateral radiograph demon-

H. K. Graham, FRCS. Senior Orthopaedic Registrar

The Fracture Clinic. Royal Victoria Hospital, Belfast BT12 6BA. Northern Ireland.

G. F. McCoy, FRCS. Senior Orthopaedic Registrar

R. A. B. Mollan, FRCS, Professor of Orthopaedic Surgery

Department of Orthopaedic Surgery. The Queen's University of Belfast, Musgrave Park Hospital, Belfast BT9 $7 \mathrm{JB}$. Northern Ireland. Requests for reprints should be sent to Mr H. K. Graham.

( 1985 British Editorial Society of Bone and Joint Surgery $0301-620 \mathrm{X} / 85 / 2046 \$ 2.00$ strated no volar or dorsal displacement of the ulna with respect to the radius (Fig. 2).

Manipulation under general anaesthesia was performed; a pronation force was applied and was increased until the radius unlocked with an audible and palpable click. This restored full, stable rotation of the forearm. A check radiograph in pronation showed the ulnar styloid in its normal anatomical position, but with an undisplaced fracture through its base (Fig. 3). The wrist was immobilised in a long-arm plaster cast with the elbow flexed to 90 and the forearm pronated. The cast was removed after six weeks and vigorous physiotherapy instituted. Flexion and extension of the wrist gradually returned, but forearm rotation remained restricted. At review, one year after injury, pronation was 60 , but supination only 20 . There was some mild pain present on the ulnar side of the wrist, but no instability or clicking.

\section{ANATOMY}

Cadaveric studies were performed in an attempt to understand the mechanism of injury and to define the pathological anatomy. In the first study, a partially dissected fresh cadaveric wrist was forcefully rotated until a locked position at 180 of supination was achieved. Dissection of the volar aspect of the wrist was then completed. The pronator quadratus had sustained a complete tear through its substance, with a $1.5 \mathrm{~cm}$ gap between the ends. The triangular fibrocartilage (articular disc) had been torn from its radial attachment (Figs 4 and 5). Part of the anterior radio-ulnar ligament and joint capsule remained intact, but had prolapsed across the articular surface of the distal ulna.

To determine more fully the role of the articular disc and the contribution to stability made by the radio-ulnar ligaments, a second dissection was carried out. After complete section of the anterior capsule and of the anterior radio-ulnar ligaments, a hypersupination force ruptured the articular disc and then produced the more usual volar dislocation of the ulna (Fig. 6c). If some anterior fibres were left intact, the radius could be locked onto the ulnar styloid without volar displacement of the latter (that is, the injury was purely rotational, as seen in Figure 6d).

In a further study using two preserved cadavers, the 


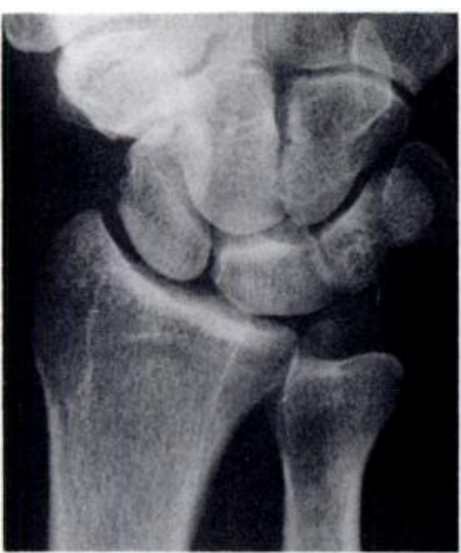

Fig. 1

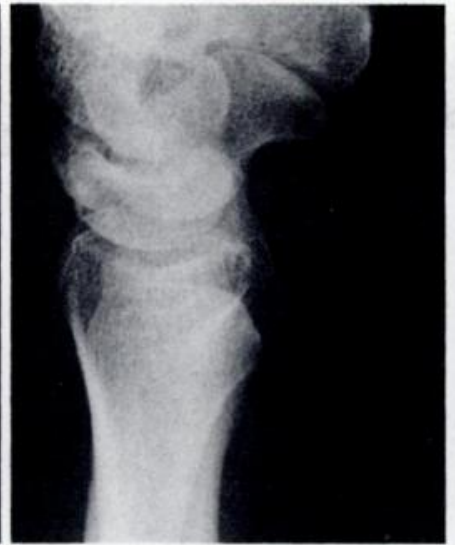

Fig. 2

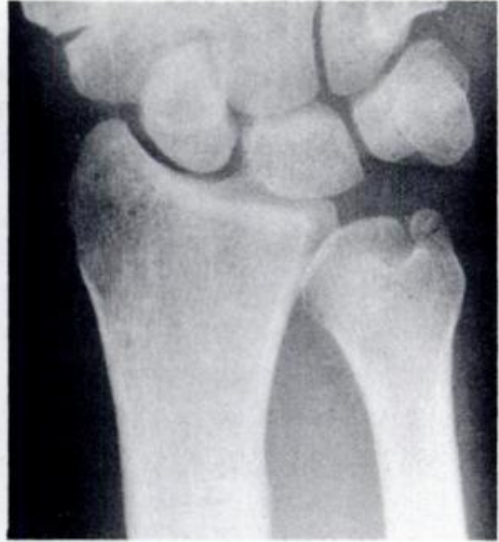

Fig. 3

Hypersupination injury. Figure 1-Postero-anterior view of right wrist. This view is normally taken with the wrist in pronation, when the styloid process of the ulna is seen on the side of the ulna away from the radius. Here it is against the radius. Figure 2-A true lateral view shows no volar or dorsal displacement of the ulna. Figure 3-Postero-anterior view (ie in pronation) showing the position after reduction: in this view, the one usually taken for the wrist, the styloid is back in its normal position.

hands were completely removed through the radiocarpal joint. This gave an excellent "end-on" view of the articular disc and the radio-ulnar ligaments, which were inspected while rotational forces were applied. The radio-ulnar ligaments were noted to be firmly attached to the peripheral rim of the articular disc. However, when they were repeatedly stressed to near breaking point, they formed a distinct raised band and tended to separate from the synovium-lined articular disc. It was impossible to reproduce a purely rotational injury in these specimens, almost certainly because soft-tissue changes had occurred as a result of preservation.

\section{DISCUSSION}

Isolated injury to the distal radio-ulnar joint is well recognised but rare. Several authors have described volar dislocation from hypersupination injuries and dorsal dislocation from pronation injuries. In many instances, the forearm becomes "locked" with respect to rotation. Other causes of locking, such as the presence of a loose body, have also been reported (Michaelis 1940). 'Typical causes of these rotational injuries with dislocation include falls on the outstretched hand, injuries from cranking handles, and carrying heavy weights in the extended supinated hand (Rose-Innes 1960).

If volar dislocation is suspected, a true lateral radiograph in neutral rotation will show the ulnar displacement. Obtaining such a film may, however, be difficult if the wrist is too painful to position correctly. If any doubt exists, a single CT scan through the distal radio-ulnar joint will resolve the issue (Mino, Palmer, Levinsohn 1983). The pure rotational injury, which we describe, is best appreciated on an anteroposterior radiograph.

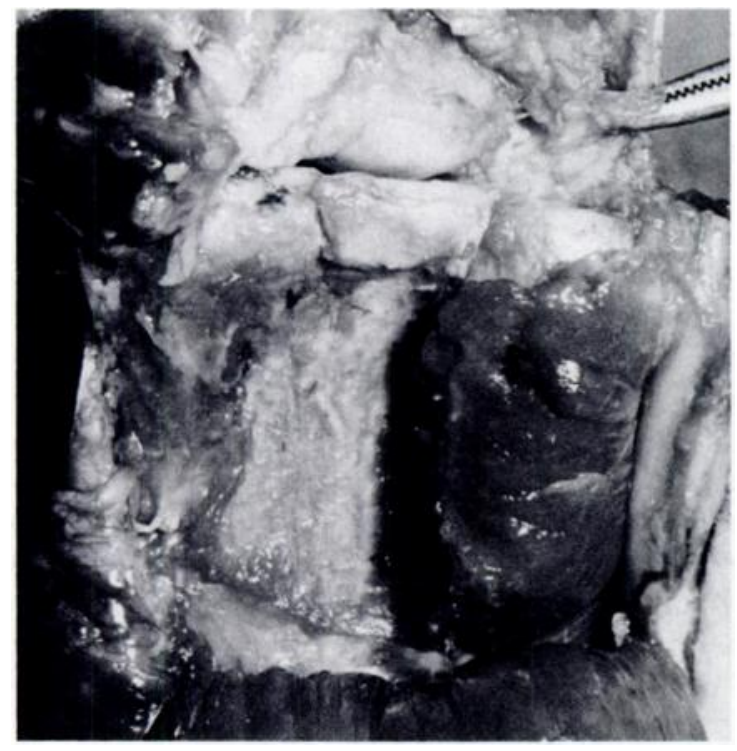

Fig. 4

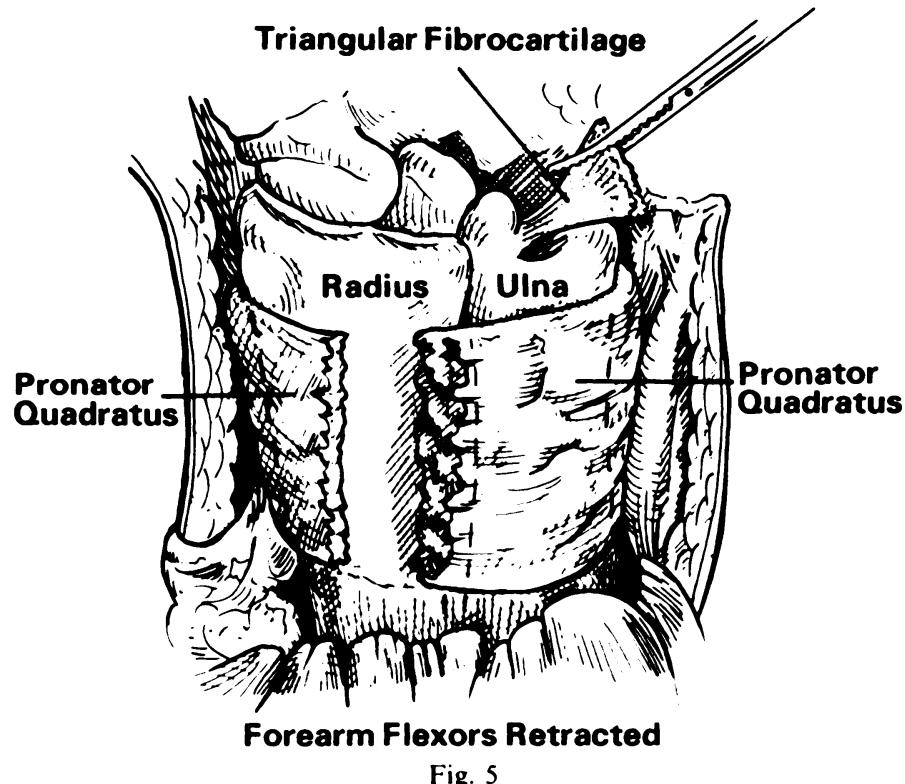

Fig. 5

Photograph and diagram of a cadaveric dissection showing complete disruption of the pronator quadratus. 


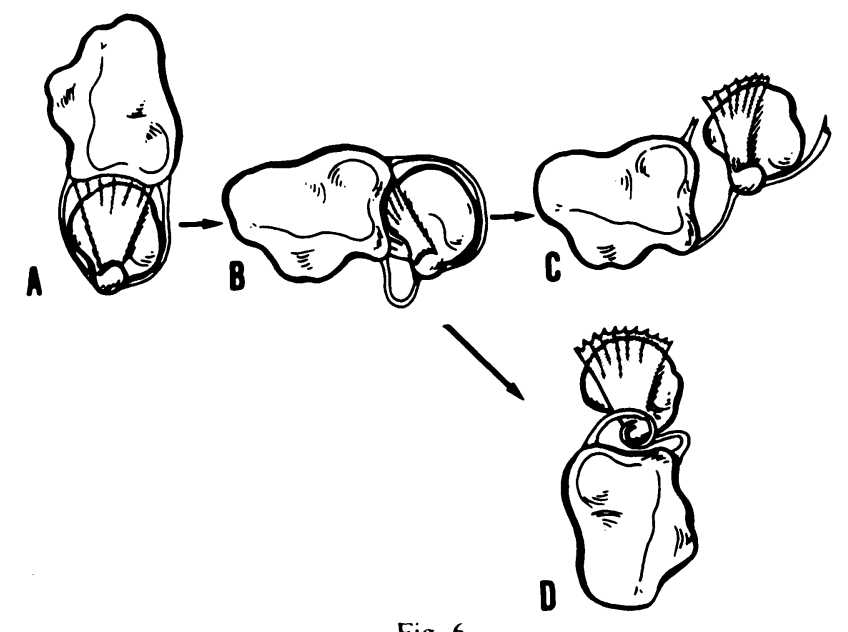

Fig. 6

Diagrams of the radius and ulna with the articular disc and radio-ulnar ligaments shown as separate structures for clarity. $(A)$, relative position of these structures in neutral rotation: $(B)$, relative position in maximal supination $(90)$ : $(C)$. the usual hypersupination injury, ie volar subluxation of the ulna with rupture of the anterior radio-ulnar ligaments and of the articular disc: and $(D)$. locked hypersupination at 180 . as discussed in the case report and demonstrated by dissections.

As with any joint, stability is governed by bony configuration, augmented by muscle and ligament attachment. The sigmoid notch of the radius is shallow, and provides the distal radio-ulnar joint with little intrinsic stability, considering the range of movements required. The pronator quadratus is ideally placed to stabilise the joint, especially in supination when its fibres are tightest (Johnson and Shrewsbury 1976). A hypersupination injury may produce a complete tear of the substance of this muscle, as we have shown. Subsequent fibrosis and contracture at this site is considered by some to be responsible for restricted rotation after immobilisation (Pezeshki and Weiland 1978). A policy of immobilisation in neutral rotation may be better in terms of preserving rotational movements, so long as the dislocation is fully reduced.

Palmer and Werner (1981) have given a complete account of the ligamentous structures stabilising the distal radio-ulnar joint. They have coined the term triangular fibrocartilage complex (TFCC) to describe the structures referred to by previous authors under different names. They consider the components of the TFCC to be the triangular fibrocartilage itself (the articular disc), the meniscus homologue, the ulnar collateral ligament, the radio-ulnar ligaments, and the sheath of the extensor carpi ulnaris. They emphasised the homogeneous nature of these structures, the named parts being merely anatomical portions of a continuous whole. They also demonstrated two important functions of the TFCC stabilisation of the distal radio-ulnar joint and the provision of a cushion for the ulnar carpus.

In dislocations of the distal radio-ulnar joint various parts of the TFCC may be torn. Coleman in 1960 reported good early results after excision of the articular disc for traumatic tears. However, the close anatomical relationship between the central (articular) disc and the radio-ulnar ligaments makes removal of one impossible without damage to the other. Palmer and Werner (1981) strongly advised against excision of the disc because of the likelihood of instability. In any case, the articular disc is prone to degeneration with age and has a reported incidence of central perforation ranging from $30 \%$ to 53\% (Albert. Wohl and Rechtman 1963; Palmer and Werner 1981). The significance of perforation on a posttraumatic arthrogram must therefore be carefully considered before advising operative treatment (Palmer, Levinsohn and Kuzma 1983).

Although in the normal wrist the triangular fibrocartilage and the radio-ulnar ligaments are in continuity, we suggest from our studies that various patterns of rupture may occur in rotation injuries. If all the anterior structures are torn in hypersupination, the usual volar dislocation will result (Fig. 6c). If some fibres remain in continuity, sufficient volar restraint may exist to achieve the purely rotational injury described. We therefore suggest that the radio-ulnar ligaments have a contributory role in stabilising the distal radio-ulnar joint. Clinicians should be aware of these rare injuries when a patient with a painful wrist gives a history of a rotation injury.

The authors wish to thank Mr Brendan Ellis and Mr Roy Creighton for their excellent photography and illustrations, and Miss Lily McGuffin for typing the manuscript.

\section{REFERENCES}

Albert SM, Wohl MA, Rechtman AM. Treatment of the disrupted radio-ulnar joint. $J$ Bone Joint Surg $[A m]$ 1963;45-A:1373-81.

Coleman HM. Injuries of the articular disc at the wrist. $J$ Bone Joint Surg [Br] 1960:42-B:522-9.

Dobyns JH, Linscheid RL. Fractures and dislocations of the wrist. In: Rockwood CA Jr. Green DP, eds. Fractures. Philadelphia: JB Lippincott. 1975:1:380-5.

Gibson A. Uncomplicated dislocation of the inferior radio-ulnar joint. $J$ Bone Joint Surg 1925:7:180-9.

Johnson RK, Shrewsbury MM. The pronator quadratus in motions and in stabilization of the radius and ulna at the distal radioulnar joint. $J$ Hand Surg 1976:1:205-9.

Michaelis LS. Locking wrist. Lancet 1940;ii:229-30.

Mino DE, Palmer AK, Levinsohn EM. The role of radiography and computerized tomography in the diagnosis of subluxation and dislocation of the distal radioulnar joint. $J$ Hand Surg 1983:8 23-31.
Morrissy RT, Nalebuff EA. Dislocation of the distal radioulnar joint: anatomy and clues to prompt diagnosis. Clin Orthop 1979:144:154-8.

Palmer AK, Werner FW. The triangular fibrocartilage complex of the wrist: anatomy and function. $J$ Hand Surg 1981:6:153-62.

Palmer AK, Levinsohn EM, Kuzma GR. Arthrography of the wrist. $J$ Hand Surg 1983:8: 15-23.

Pezeshki CM, Weiland AJ. Bilateral dorsal dislocation of the distal radio-ulnar joint. J Trauma 1978:18:673-6.

Rose-Innes AP. Anterior dislocation of the ulna at the inferior radioulnar joint: case reports, with a discussion of the anatomy of rotation of the forearm. J Bone Joint Surg $[B r] 1960 ; 42-B: 515-21$.

Vesely DG. The distal radio-ulnar joint. Clin Orthop 1967:51 : 75-91.

Wright PE. Dislocations. In: Edmonson AS. Crenshaw AH, eds. Camphell's operative orthopaedics. St Louis: CV Mosby. 1980:447-9. 